\title{
A Retrospective Analysis of Psychiatric Presurgical Evaluation of Children and Adolescents Evaluated for Epilepsy Surgery in a Comprehensive Epilepsy Care Unit of Mumbai
}

\author{
${ }^{1}$ Department of Psychiatry, Seth Gordhandas Sunderdas Medical \\ College and King Edward Memorial Hospital, Mumbai, Maharashtra, \\ India \\ 2 Department of Psychiatry, Lokmanya Tilak Municipal Medical \\ College and Lokmanya Tilak Municipal General Hospital, Mumbai, \\ Maharashtra, India \\ 3 Department of Neurology, Seth Gordhandas Sunderdas Medical \\ College and King Edward Memorial Hospital, Mumbai, Maharashtra, \\ India
}

Neena S. Sawant ${ }^{1}$ Suraj S. Singh ${ }^{1}$ Sachin Mahajan ${ }^{2}$ Sangeeta H. Ravat ${ }^{3}$

J Neurosci Rural Pract 2022;13:95-100.
Address for correspondence Neena S. Sawant, MD, DPM, PGDHHM, PGDMLS, Department of Psychiatry, Seth Gordhandas Sunderdas Medical College and King Edward Memorial Hospital, Parel, Mumbai 400012, Maharashtra, India (e-mail: drneenas@yahoo.com).

\section{Abstract \\ Keywords \\ - epilepsy \\ - children with epilepsy \\ - psychiatric disorders \\ - presurgical evaluation \\ - refractory epilepsy}

Background Epilepsy being one of the most prevalent neurological diseases in children is associated with psychopathology and academic concerns. Epilepsy surgery is considered for refractory epilepsy at some centers in India and hence this study was undertaken to find out prevalence and type of psychopathology in children and adolescents with refractory epilepsy before epilepsy surgery.

Methods All data were analyzed from the records of patients undergoing preepilepsy surgery protocol workup in comprehensive center of epilepsy care at a general municipal hospital in Mumbai. A record of 150 children and adolescents in the age group of 3 to 18 years over a period of 10 years was taken and all details of demographics, epilepsy, and psychopathology were recorded.

Results The mean age for our sample was $11.4 \pm 3.4$ years and a male preponderance was seen. Majority $(80 \%)$ of the children were pursuing education. The duration of seizure disorder was approximately $4.41+2.36$ years and complex partial seizures were seen commonly in $50 \%$ of the children. Both magnetic resonance imaging (MRI) and video electroencephalography (VEEG) findings revealed right sided lateralization followed by left in majority of the patients. Psychopathology was seen in 70 (46\%) patients with mental retardation, hyperkinetic disorders affecting attention and activity and oppositional defiant disorder, and unspecified mental disorder due to underlying brain damage being the type of International Classification of Disease-10th Revision (ICD-10) disorders seen. Patients with psychopathology showed a left-sided published online January 11,2022
DOI https://doi.org/ 10.1055/s-0041-1742159. ISSN 0976-3147. (c) 2022. Association for Helping Neurosurgical Sick People. All rights reserved.

This is an open access article published by Thieme under the terms of the Creative Commons Attribution-NonDerivative-NonCommercial-License, permitting copying and reproduction so long as the original work is given appropriate credit. Contents may not be used for commercial purposes, or adapted, remixed, transformed or built upon. (https://creativecommons.org/ licenses/by-nc-nd/4.0/)

Thieme Medical and Scientific Publishers Pvt. Ltd., A-12, 2nd Floor, Sector 2, Noida-201301 UP, India 
predominance on their MRI and VEEG findings for laterality of the epileptogenic focus as compared with right side.

Conclusion Refractory seizures and associated psychopathology impact family life, friendships, and academics and worsen prognosis and quality of life. Screening for psychopathology in children with epilepsy would therefore lead to better outcomes especially prior to epilepsy surgery.

\section{Introduction}

Among the common neurological conditions affecting children and adolescents, epilepsy is the forerunner, with a greater burden seen in developing countries, ${ }^{1}$ with an estimated prevalence of 5.5 cases per 1,000 children, ${ }^{2,3}$ Many of these cases would also be having refractory epilepsy/seizures with associated psychiatric problems. ${ }^{1}$ The International League Against Epilepsy (ILAE) defines drug-resistant/refractory seizures as "failure of adequate trials of two tolerated and appropriately chosen antiepileptic schedules (whether as monotherapies or in combination) to achieve seizure freedom." $^{2}$

Comorbid psychopathology is seen very commonly in children with epilepsy (CWE) and its prevalence is around 16 to $77 \%{ }^{3}$ Most commonly seen psychiatric problems include cognitive impairments, academic concerns, and other neurobehavioral disorders. ${ }^{4}$

Multiple etiological factors, like frequency of seizures, refractoriness, prenatal or postnatal insult, early age of onset, use of multiple antiepileptic medications, or symptomatic epilepsy, can result in psychiatric disorders in epilepsy. ${ }^{5-8}$ The various behavioral psychiatric disorders seen in children include hyperactivity, impulsivity, attention deficit, conduct problems, defiant behavior, lying, stealing, and others. ${ }^{4}$ Most of the evidences of psychiatric disorders in CWE come from studies done in developed countries, whereas there is a dearth of literature from developing countries. Treating pediatricians or neurologists may not be aware of the underlying behavioral issues and parents may not report the same, as there is so much stigma attached to epilepsy itself. Resolution of the epilepsy does not necessarily lead to resolution of the psychopathology. Therefore, a separate and thorough psychiatric assessment and treatment is essential in these patients.

Researchers have found a relation between structural foci laterality and interictal psychopathology in patients evaluated for epilepsy surgery. Left-sided foci are associated with anxiety, while the right-sided foci have more of depressive symptoms associated with them in patients of temporal lobe seizures. ${ }^{9,10}$ Lindsay et al have found increased psychopathology in children with left sided focal seizures. ${ }^{11}$ However, this finding is not consistent with other studies. ${ }^{12-14}$

Epilepsy surgery is the treatment offered for refractory epilepsy in a few institutes in India and includes a comprehensive, thorough, and multidimensional approach at our center. There is very little Indian data on the psychopatholo- gy prevalent in children and adolescents prior to undergoing epilepsy surgery. Hence, we decided to analyze a10-year-old data of our center, for the prevalence and type of psychopathology and the association of lateralization of epileptic focus with psychopathology in those children and adolescents undergoing epilepsy surgery.

\section{Materials and Methods}

The Comprehensive Epilepsy Care Centre of the Department of Neurology evaluates 10 to 12 patients every month for epilepsy surgery. The team consists of neurologists, neuropsychologists, psychiatrist, neurosurgeon, nuclear medicine specialist, and radiologist. Each patient undergoes neurodiagnostic evaluation with video electroencephalography (VEEG) epilepsy protocol (72 hours), magnetic resonance imaging (MRI) brain epilepsy protocol, and neuropsychological testing with a detailed psychiatric evaluation. Patients diagnosed by the consultant neurologist as refractory epilepsy are assessed for presurgical evaluation. Patients are referred to the psychiatrist for detailed assessment and fitness for epilepsy surgery is given if there is no underlying psychopathology. If patients are found to be having any psychopathology, they are treated and followed-up regularly over 10 to 12 weeks. Fitness for epilepsy surgery is only given after improvement in behavioral and emotional symptoms. The details of each patient are recorded in the pre epilepsy surgery proforma which includes the demographic variables as follows: age, sex, handedness, education status, detailed neurological findings like type of seizure, duration of seizure, associated automatism, number of antiepileptics, VEEG findings, MRI brain findings, lateralization of epileptogenic focus along with details of psychiatric assessment with diagnosis given on International Classification of Disease-10th Revision (ICD-10) diagnostic guidelines, ${ }^{15}$ and a detailed neuropsychological assessment report with intelligence quotient (IQ) testing and memory profile.

Comprehensive epilepsy care program is in practice from the year 2001 onward in the General Municipal Hospital. This was a retrospective study conducted after institutional ethics committee approval. Data of children and adolescents less than 18 years of age was collected from the past 10 years' preepilepsy surgery proformas maintained in the department of neurology. Around 220 child and adolescent patients were evaluated for epilepsy surgery. In the present study, we included only completely filled proformas of those children and adolescents who were in the age range of 3 to 18years of 
age, had been diagnosed as refractory epilepsy due to any cause and were referred for epilepsy surgery workup. Children who had received psychiatric treatment before or were on psychiatric treatment were excluded from the data analysis. After satisfying the inclusion and exclusion criteria of the 220 patients, 70 proformas with incomplete information or previous psychiatric problems were discarded and the data of 150 completely filled proformas were analyzed. All the patients in the epilepsy surgery program were diagnosed for psychiatric disorders as per the ICD-10 diagnostic criteria by the consultant psychiatrist who is the part of the multidisciplinary team. The child and adolescent patients are then asked to follow-up separately in the child guidance clinic of the department of psychiatry for further evaluation and management and are given fitness for epilepsy surgery after improvement in their symptoms. The patients who are considered eligible for epilepsy surgery are then discussed after all the above evaluations in a joint meeting held with the entire comprehensive epilepsy care unit to decide the type of epilepsy surgery and all the information is documented in the proformas.

\section{Statistical Analysis}

Demographic variables, details of seizure type, frequency and duration, MRI findings, lateralization details, and prevalence and type of psychopathology were all assessed using frequency distribution.

\section{Results}

The mean age of our sample was $11.4 \pm 3.4$ years and a male preponderance was seen. Majority (80\%) of the children were pursuing education. Nearly two-thirds of them were right handed. The duration of seizure disorder was $4.41 \pm 2.36$ years. Complex partial seizures were seen in $50 \%$ of the children followed by generalized seizures (34\%) and myoclonic seizures (15\%). Daily seizures were seen in 25 (16.6\%) patients with up to five seizures per month in approximately 55 (36.6\%) children. More than five seizures occurring over 3 months was the frequency in 65 (43.3\%) children. Majority of the children and adolescents had a structural lesion on their MRI with mesial temporal sclerosis (MTS) followed by focal cortical dysplasia/gliosis being the most common. Normal MRI was seen in only $3(2 \%)$ patients (-Table $\mathbf{1}$ ).

Both MRI and VEEG findings revealed right-sided lateralization followed by left in majority of the patients. Normal MRI was seen in only one patient, whereas there was no seizure activity seen in the VEEG of three patients ( - Table 2 ).

Psychopathology was present in $70(46 \%)$ children and absent in the remaining 80 (54\%) children. The diagnosis of the psychiatric disorders as per the ICD-10 criteria of these 70 children revealed mental retardation in $22(31 \%)$ patients and hyperkinetic disorders affecting attention and activity in $20(28 \%)$ children. The other conditions like oppositional defiant disorder or conduct disorder were seen in a less number of patients. None of our sample had any emotional disorders or anxiety which is usually seen in CWE ( - Table 3 ).
Table 1 Demographic and seizure details

\begin{tabular}{|c|c|}
\hline Variables & $\begin{array}{l}\text { Number of patients } \\
(n=150) \\
\text { Mean } \pm S D / n(\%)\end{array}$ \\
\hline Age (y) & $11.4 \pm 3.49$ \\
\hline \multicolumn{2}{|l|}{ Sex } \\
\hline Male & $93(62)$ \\
\hline Female & $57(38)$ \\
\hline \multicolumn{2}{|l|}{ Education } \\
\hline Illiterate & $28(18.6)$ \\
\hline Primary & $35(23.3)$ \\
\hline Secondary & $77(51.3)$ \\
\hline Higher secondary & $10(6.6)$ \\
\hline \multicolumn{2}{|l|}{ Handedness } \\
\hline Right & $103(68.6)$ \\
\hline Left & $47(31.3)$ \\
\hline Duration of seizure & $4.41+2.36$ years \\
\hline \multicolumn{2}{|l|}{ Type of seizure } \\
\hline Generalised seizures & $52(34.6)$ \\
\hline Complex partial seizures & $75(50)$ \\
\hline Myoclonic seizures & $23(15.3)$ \\
\hline \multicolumn{2}{|l|}{ Frequency of seizure } \\
\hline Daily & $25(16.6)$ \\
\hline Up to 5 per month & $55(36.6)$ \\
\hline$>5$ in 3 months & $65(43.3)$ \\
\hline \multicolumn{2}{|l|}{ MRI findings } \\
\hline Normal & $3(2)$ \\
\hline MTS & $67(44.6)$ \\
\hline Gliosis/dysplasia & $34(22.6)$ \\
\hline Tumor (cyst/DNET) & $33(22)$ \\
\hline $\begin{array}{l}\text { Other(encephalomalacia / } \\
\text { perinatal insult/encephalitis) }\end{array}$ & $13(8.6)$ \\
\hline
\end{tabular}

Abbreviations: DNET, dysembryoplastic neuroepithelial tumor; MRI, magnetic resonance imaging; SD, standard deviation.

When patients having psychopathology $(n=70)$ were assessed on their MRI and VEEG findings for laterality of the epileptogenic focus then $37(52.8 \%)$ patients had a leftsided predominance as compared to 23 (32.5\%) patients who had right side. About 10 (14.2\%) patients had a bilateral focus. However, we did not find any specific pattern of psychopathology in those having left or right focus. The mental retardation and behavioral problems were more or less equally seen in both-sided lesions.

\section{Discussion}

Epilepsy is one of the commonest neurological disorders affecting children and has a prevalence of 5.5 cases per 1,000 children in developing countries. ${ }^{1,16,17}$ There is bimodal 
Table 2 Lateralization as per MRI and video EEG findings

\begin{tabular}{|l|l|l|l|}
\hline \multicolumn{2}{|l|}{ MRI findings } & \multicolumn{2}{|l|}{ Video EEG findings } \\
\hline $\begin{array}{l}\text { Lateralization } \\
\text { as per MRI }\end{array}$ & $\begin{array}{l}\text { Number } \\
\text { of patients } \\
(\boldsymbol{n}=\mathbf{1 5 0 )} \\
\boldsymbol{n ( \% )}\end{array}$ & $\begin{array}{l}\text { Lateralization } \\
\text { as per video } \\
\text { EEG }\end{array}$ & $\begin{array}{l}\text { Number of } \\
\text { patients } \\
(\boldsymbol{n}=150) \\
\boldsymbol{n}(\%)\end{array}$ \\
\hline Right & $68(45.3)$ & Right & $64(42.6)$ \\
\hline Left & $64(42.6)$ & Left & $53(35.3)$ \\
\hline Bilateral & $10(6.6)$ & Bilateral & $26(17.3)$ \\
\hline Diffuse & $7(4.6)$ & Generalized & $4(2.6)$ \\
\hline Normal & $1(0.6)$ & $\begin{array}{l}\text { No epileptiform } \\
\text { activity }\end{array}$ & $3(2)$ \\
\hline
\end{tabular}

Abbreviations: EEG, electroencephalogram; MRI, magnetic resonance imaging.

Table 3 Type of psychopathology as per ICD10 criteria

\begin{tabular}{|l|l|}
\hline Type of psychopathology & $\begin{array}{l}\text { Number of } \\
\text { patients }(\boldsymbol{n}=70) \\
\boldsymbol{n}(\%)\end{array}$ \\
\hline Mental retardation & $22(31.4)$ \\
\hline $\begin{array}{l}\text { Hyperkinetic disorder: disorder of } \\
\text { attention and activity }\end{array}$ & $20(28.8)$ \\
\hline Conduct disorder unspecified & $3(4.2)$ \\
\hline Oppositional defiant disorder & $10(14.2)$ \\
\hline $\begin{array}{l}\text { Unspecified mental disorder due to } \\
\text { brain damage and dysfunction and to } \\
\text { physical disease }\end{array}$ & $15(21.4)$ \\
\hline
\end{tabular}

Abbreviation: ICD-10, International Classification of Disease-10th Revision.

distribution of age for incidence and prevalence of epilepsy, that is, one in children and another in old age. ${ }^{18,19}$ Several researchers have reported that onset of epilepsy is usually increased in the age group of 5 and 10 years, ${ }^{20,21}$ and our findings are keeping in those of Shinnar and Pellock ${ }^{22}$ where children between the ages of 5 and 14 years had active epilepsy. Gender distribution has revealed a male preponderance as reported by Hauser et $\mathrm{al}^{20}$ and Christensen et $\mathrm{al}^{23}$ who reported epilepsy to be slightly more prevalent in males than females.

Moreover, 28(\%) of our children were illiterate and never attended school while the others were pursuing primary and secondary education. Epilepsy is known to affect schooling and many children do drop out of school due to the refractoriness of the seizure. ${ }^{24}$ However, we did not find any school drop outs among our sample. Nearly two-thirds of the sample was right handed with only 47 patients being left handed. There have been several studies reporting atypical pattern of handedness in patients with unilateral mesial temporal lobe epilepsy (MTLE) especially those with leftsided MTLE, ${ }^{25}$ and this was associated with early age at epilepsy onset where failed surgical outcomes were due to false lateralization of the major hemisphere. ${ }^{26}$ Indian researchers have reported certain risk factors for refractory seizures in children which include partial seizures, presence of neurological deficits, history of brain insults with evidence of structural brain damage, febrile seizures, presence of increased seizure frequency of more than one per month with a poor or no response to the first antiepileptic drug. ${ }^{27,28}$ A total of 75 children had complex partial seizures and 52 had generalized seizures which are in keeping with the common seizure types seen in children. The MRI findings revealed structural lesions like MTS which was most common, followed by dysplasia/gliosis and tumors. Udani et al have postulated that perinatal insults constituted $50 \%$ of symptomatic epilepsies which usually have an onset in the early 3 years of life and later these structural lesions lead to refractory seizures. ${ }^{29,30}$ However, in our sample, there were few patients with perinatal ischemia or hypoxic brain damage.

When all the children were assessed for presence of psychopathology as per ICD-10 diagnostic criteria, then 70 (46\%) patients had psychopathology. Hoare reported psychiatric disturbance in $48 \%$ of $\mathrm{CWE}^{31}$ which is in keeping with our findings. A slightly lower prevalence of 26 to $41 \%$ of psychiatric that comorbidities in CWE has been reported by some researchers. ${ }^{32}$

Udani et al have reported the prevalence of psychopathology in CWE to be ranging from 16 to $77 \%{ }^{4}$ This high prevalence is due to various factors like seizure variables, duration, frequency and type of seizure, poor response, antiepileptic medication, as well the underlying structural changes.

The various psychiatric disorders seen in descending order of frequency in our patients included mental retardation, hyperkinetic disorders with disturbance of activity and attention, behavioral change due to underlying brain damage, oppositional defiant disorders, and conduct disorders. Austin in his study stated that around $41.1 \%$ of CWE were at risk for both internalizing and externalizing behavior problems. ${ }^{33}$ Several researchers found a prevalence of attentiondeficit hyperactivity disorder (ADHD) to be 30 to $40 \%$ in CWE which is much higher than in general population. ${ }^{34-36}$ Jones et al reported that $26.4 \%$ of CWE without developmental and other neurological conditions had hyperactivi$\mathrm{ty}^{32}$ whereas in CWE, the prevalence of ADHD was approximately $70 \%{ }^{37}$ Our findings are in keeping with those of above researchers where the possible causes of hyperkinetic disorders in CWE could be the underlying mechanism causing conditions, associated underlying structural brain damage, and adverse effects of antiepileptics. ${ }^{38}$ Also nearly 15 patients had behavioral disturbances which were due to the underlying structural brain damage. Psychopathology can occur prior to the onset of epilepsy or as its sequelae due to the interplay between several multifactorial etiologies like vulnerability, genetic susceptibility, ante- or postnatal complications, developmental delays, and the biochemical and neurophysiologic effects in the preictal, ictal, or postictal phases. Jones et al had found the prevalence of oppositional defiant disorder (ODD) to be between 10 and $17 \% 32$ which is in keeping with our 
findings. Thomas-Souza et $\mathrm{al}^{34}$ also reported the prevalence of ODD to be around $11.1 \%$.

We did find mental retardation in 22 (31\%) children. Reilly et al in their population-based study found intellectual disability as a common comorbidity in CWE with a prevalence between 30 and $40 \%$ which is in keeping with our findings. ${ }^{39}$ CWE have below average or borderline intellectual functioning due to the kindling phenomena, refractoriness of seizure, or cognitive slowing due to the antiepileptic medications. ${ }^{40}$ Most of the children with refractory seizures are also on more than two antiepileptics which increases the side effects.

We did find a predominant left-sided lateralization in the patients who had psychopathology as compared with those having right or bilateral lesions, though we did not associate the type of psychopathology to laterality, as we had only externalizing behaviors in our sample. Potegal et al found that right foci were associated with excessive anger and left foci with excessive fear. ${ }^{41}$

\section{Conclusion}

The study helps us to know the coexisting psychopathology in children and adolescents of epilepsy undergoing epilepsy surgery and the need to have a thorough psychiatric workup of all patients undergoing epilepsy surgery. Early diagnosis will result in prompt treatment of the coexisting psychopathology; and hence, it is necessary that a psychiatrist should be a part of the multidisciplinary team to improve the overall quality of life of the patients and their families with treatment of the underlying psychiatric problems. A liaison between pediatrician, neurologist, and psychiatrist will definitely help in the prognosis of not only the seizure disorder but also the behavioral and emotional disturbances seen in pediatric epilepsy patients.

\section{Ethical Approval}

The study was assessed and approved by the institutional ethics committee/institutional review board and that the letter of approval is available with us for examination. The ethical aspects of the study were conforming to the Declaration of Helsinki.

\section{Funding}

None.

\section{Conflict of Interest}

None declared.

\section{References}

1 Bujoreanu IS, Ibeziako P, Demaso DR. Psychiatric concerns in pediatric epilepsy. Pediatr Clin North Am 2011;58(04):973-988 , xii

2 Kwan P, Arzimanoglou A, Berg AT, et al. Definition of drug resistant epilepsy: consensus proposal by the ad hoc Task Force of the ILAE Commission on Therapeutic Strategies. Epilepsia 2010;51(06): 1069-1077

3 Udani V. Pediatric epilepsy-an Indian perspective. Indian J Pediatr 2005;72(04):309-313
4 Plioplys S, Dunn DW, Caplan R. 10-year research update review: psychiatric problems in children with epilepsy. J Am Acad Child Adolesc Psychiatry 2007;46(11):1389-1402

5 Høie B, Sommerfelt K, Waaler PE, Alsaker FD, Skeidsvoll H, Mykletun A. Psychosocial problems and seizure-related factors in children with epilepsy. Dev Med Child Neurol 2006;48(03): 213-219

6 Besag FM. Behavioral aspects of pediatric epilepsy syndromes. Epilepsy Behav 2004;5(Suppl 1):S3-S13

7 Hanssen-Bauer K, Heyerdahl S, Eriksson AS. Mental health problems in children and adolescents referred to a national epilepsy center. Epilepsy Behav 2007;10(02):255-262

8 Choudhary A, Gulati S, Sagar R, Kabra M, Sapra S. Behavioral comorbidity in children and adolescents with epilepsy. J Clin Neurosci 2014;21(08):1337-1340

9 Altshuler LL, Devinsky O, Post RM, Theodore W. Depression, anxiety, and temporal lobe epilepsy. Laterality of focus and symptoms. Arch Neurol 1990;47(03):284-288

10 Devinsky O, D'Esposito M. Neurology of Cognitive and Behavioral Disorders. New York, NY: Oxford: University Press; 2004:451

11 Lindsay J, Ounsted C, Richards P. Long-term outcome in children with temporal lobe seizures. III: Psychiatric aspects in childhood and adult life. Dev Med Child Neurol 1979;21(05):630-636

12 Herranz JL, Armijo JA, Arteaga R. Clinical side effects of phenobarbital, primidone, phenytoin, carbamazepine, and valproate during monotherapy in children. Epilepsia 1988;29(06):794-804

13 Caplan R, Siddarth P, Gurbani S, Ott D, Sankar R, Shields WD. Psychopathology and pediatric complex partial seizures: seizurerelated, cognitive, and linguistic variables. Epilepsia 2004;45(10): 1273-1281

14 Caplan R, Siddarth P, Gurbani S, Hanson R, Sankar R, Shields WD. Depression and anxiety disorders in pediatric epilepsy. Epilepsia 2005;46(05):720-730

15 The ICD-10 Classification of Mental and Behavioural Disorders. Accessed December 21, 2021 at: https://www.who.int/classifications/ icd/en/bluebook.pdf

16 Andermann F. Temporal pole and mesiotemporal epilepsy: introductory remarks. Epileptic Disord 2002;4(1, suppl 1)S7-S8

17 Carpio A, Hauser WA. Epilepsy in the developing world. Curr Neurol Neurosci Rep 2009;9(04):319-326

18 Huang M, Hong Z, Zeng J, Rong X, Sheng Y, Lu C. The prevalence of epilepsy in rural Jinshan in Shanghai [in Chinese]. Zhonghua Liu Xing Bing Xue Za Zhi 2002;23(05):345-346

19 Tran DS, Odermatt P, Le TO, et al. Prevalence of epilepsy in a rural district of central Lao PDR. Neuroepidemiology 2006;26(04):199-206

20 Hauser WA, Annegers JF, Kurland LT. Prevalence of epilepsy in Rochester, Minnesota: 1940-1980. Epilepsia 1991;32(04):429-445

21 Murphy CC, Trevathan E, Yeargin-Allsopp M. Prevalence of epilepsy and epileptic seizures in 10-year-old children: results from the Metropolitan Atlanta Developmental Disabilities Study. Epilepsia 1995;36(09):866-872

22 Shinnar S, Pellock JM. Update on the epidemiology and prognosis of pediatric epilepsy. J Child Neurol 2002;17(Suppl 1):S4-S17

23 Christensen J, Vestergaard M, Pedersen MG, Pedersen CB, Olsen J, Sidenius P. Incidence and prevalence of epilepsy in Denmark. Epilepsy Res 2007;76(01):60-65

24 Dunn DW, Johnson CS, Perkins SM, et al. Academic problems in children with seizures: relationships with neuropsychological functioning and family variables during the 3 years after onset. Epilepsy Behav 2010;19(03):455-461

25 Doležalová I, Schachter S, Chrastina J, et al. Atypical handedness in mesial temporal lobe epilepsy. Epilepsy Behav 2017;72:78-81

26 Derakhshan I. Anatomy of handedness and the laterality of seizure onset: surgical implications of new understandings in motor control. Neurol Res 2005;27(07):773-779

27 Gadgil P, Udani V. Pediatric epilepsy: the Indian experience. J Pediatr Neurosci 2011;6(Suppl 1):S126-S129 
28 Tripathi M, Padhy UP, Vibha D, et al. Predictors of refractory epilepsy in north India: a case-control study. Seizure 2011;20 (10):779-783

29 Udani VP, Dharnidharka V, Nair A, Oka M. Difficult to control epilepsy in childhood-a long term study of 123 cases. Indian Pediatr 1993;30(10):1199-1206

30 Udani V, Munot P, Ursekar M, Gupta S. Neonatal hypoglycemic brain - injury a common cause of infantile onset remote symptomatic epilepsy. Indian Pediatr 2009;46(02):127-132

31 Hoare P. The development of psychiatric disorder among schoolchildren with epilepsy. Dev Med Child Neurol 1984; 26(01):3-13

32 Jones JE, Watson R, Sheth R, et al. Psychiatric comorbidity in children with new onset epilepsy. Dev Med Child Neurol 2007;49 (07):493-497

33 Austin JK, Perkins SM, Johnson CS, et al. Behavior problems in children at time of first recognized seizure and changes over the following 3 years. Epilepsy Behav 2011;21(04): 373-381

34 Thome-Souza S, Kuczynski E, Assumpção F Jr., et al. Which factors may play a pivotal role on determining the type of psychiatric disorder in children and adolescents with epilepsy? Epilepsy Behav 2004;5(06):988-994
35 Dunn DW, Kronenberger WG. Childhood epilepsy, attention problems, and ADHD: review and practical considerations. Semin Pediatr Neurol 2005;12(04):222-228

36 Besag F, Gobbi G, Caplan R, Sillanpää M, Aldenkamp A, Dunn DW. Psychiatric and behavioural disorders in children with epilepsy (ILAE task force report): epilepsy and ADHD. Epileptic Disord 2016;18(s1):S8-S15

37 Sherman EMS, Slick DJ, Connolly MB, Eyrl KL. ADHD, neurological correlates and health-related quality of life in severe pediatric epilepsy. Epilepsia 2007;48(06):1083-1091

38 Dunn DW, Besag F, Caplan R, Aldenkamp A, Gobbi G, Sillanpaa M. Psychiatric and Behavioural Disorders in Children with Epilepsy (ILAE Task Force Report): Epidemiology of psychiatric/behavioural disorder in children with epilepsy. Epileptic Disord 2016;18:2-7

39 Reilly C, Atkinson P, Das KB, et al. Neurobehavioral comorbidities in children with active epilepsy: a population-based study. Pediatrics 2014;133(06):e1586-e1593

40 Wei SH, Lee WT. Comorbidity of childhood epilepsy.J Formos Med Assoc 2015;114(11):1031-1038

41 Potegal M, Drewel EH, MacDonald JT. Tantrums, emotion reactions and their EEG correlates in childhood benign rolandic epilepsy vs. complex partial seizures: exploratory observations. Front Behav Neurosci 2018;12:40 